Neurosurg Focus 19 (6):E12, 2005

\title{
Endoscopic navigation of the fourth ventricle
}

\author{
Technical note and preliminary experience
}

\author{
Pierluigi Longatti, M.D., Luca Basaldella, M.D., Alberto Feletti, M.D., \\ Alessandro Fiorindi, M.D., AND DoMenico BilleCI, M.D.
}

Department of Neurosurgery, Treviso Regional Hospital, University of Padova, Italy

\begin{abstract}
Transaqueductal navigation of the fourth ventricle has long been considered dangerous and of no clinical relevance. After the refinement of the endoscopic technique and supported by the extensive experience gained at the authors' institution since 1994, endoscopic exploration of the fourth ventricle has been performed by the same surgeon in 54 patients. In all cases reviewed, endoscopic navigation of the fourth ventricle was successfully performed with no related neurological deficit. This preliminary experience shows the feasibility of transaqueductal navigation of the fourth ventricle, which is made possible by the use of small, flexible endoscopes in expert hands.
\end{abstract}

\section{KEY WORDS • neuroendoscopy • fourth ventricle • cerebral aqueduct • ventricle anatomy • hydrocephalus}

The transaqueductal pathway to the fourth ventricle should not be considered analogous to the Pillars of Hercules, beyond which one dares not navigate. ${ }^{10}$ On reviewing the historical experience, we noted that the very few pioneers of endoscopic approaches to the aqueductal region by a frontal route preferred rigid instruments, and that the extraordinary versatility of flexible scopes has not been fully exploited to achieve complete control of the ventricular cavities. ${ }^{3,6,7,9}$

In this study we summarize our findings in a series of 54 patients presenting with different pathological conditions in which transaqueductal navigation has been successfully performed using flexible endoscopes. This instrument has allowed us to apply this approach, not as a challenge to the status quo, but as a surprisingly similar method to those commonly used in the more usual neuroendoscopic procedures.

\section{TECHNICAL PROCEDURE}

The surgical approach is performed through a frontal bur hole $2 \mathrm{~cm}$ anterior to the coronal suture and $2 \mathrm{~cm}$ lateral to the midline. The direction of the trajectory needed to reach the ventricles is the same as that used to perform a third ventriculostomy. The frontal horn is first cannulated with a No. 14 French peel-away catheter, and through that access port a flexible endoscope is introduced. During the whole procedure, the instrument is managed with a freehand technique by using movements of advancement, flexion, extension, and rotation. In the third ventricle, the scope is directed toward the opening of the cerebral aqueduct and advanced into the ampulla and then toward the fourth ventricle.
Although it is a confined space, the cerebral aqueduct is endowed with its own compliance; ${ }^{4}$ the aqueductal channel yields at the passage of an instrument $4 \mathrm{~mm}$ in width. After the endoscope crosses the opening of the cerebral aqueduct (Fig. 1), an initial narrowing, corresponding to the superior colliculi, is recognizable, and then a more dilated space called the aqueductal ampulla is encountered..$^{1,4,10} \mathrm{~A}$ further restriction is found corresponding to the inferior colliculi. The aqueductal space then opens under the frenulum of the posterior medullary velum into the rhomboid fossa.

In the navigation of the fourth ventricle some precautions should be adopted. Once the aqueduct is cannulated, irrigation is stopped because the instrument itself occupies and shuts the passage with its own volume. Further additions of liquid could overload the fourth ventricle, which in this particular phase of the operation could become completely trapped (depending on the underlying pathological entity). Related episodes of threatening bradycardia could be the clinical consequence. ${ }^{5}$

Another observation concerns the view offered to the neurosurgeon; the posterior deflection of the scope and posterior rotation cause an upside-down view of the video endoscopic images. Therefore, it should be kept in mind that the dorsal and ventral anatomical structures are projected downward and upward, respectively, in the monitor. In that way, for instance, when the floor of the rhomboid fossa is up, the fastigium is down. Also, the tela choroidea with the affixed plexus is visible in the downward position of the videoendoscopic monitor (Fig. 2).

The sulcus medianus of the fourth ventricle is the guiding anatomical mark leading toward the posterior triangle of the rhomboid fossa, with clear evidence of calamus 


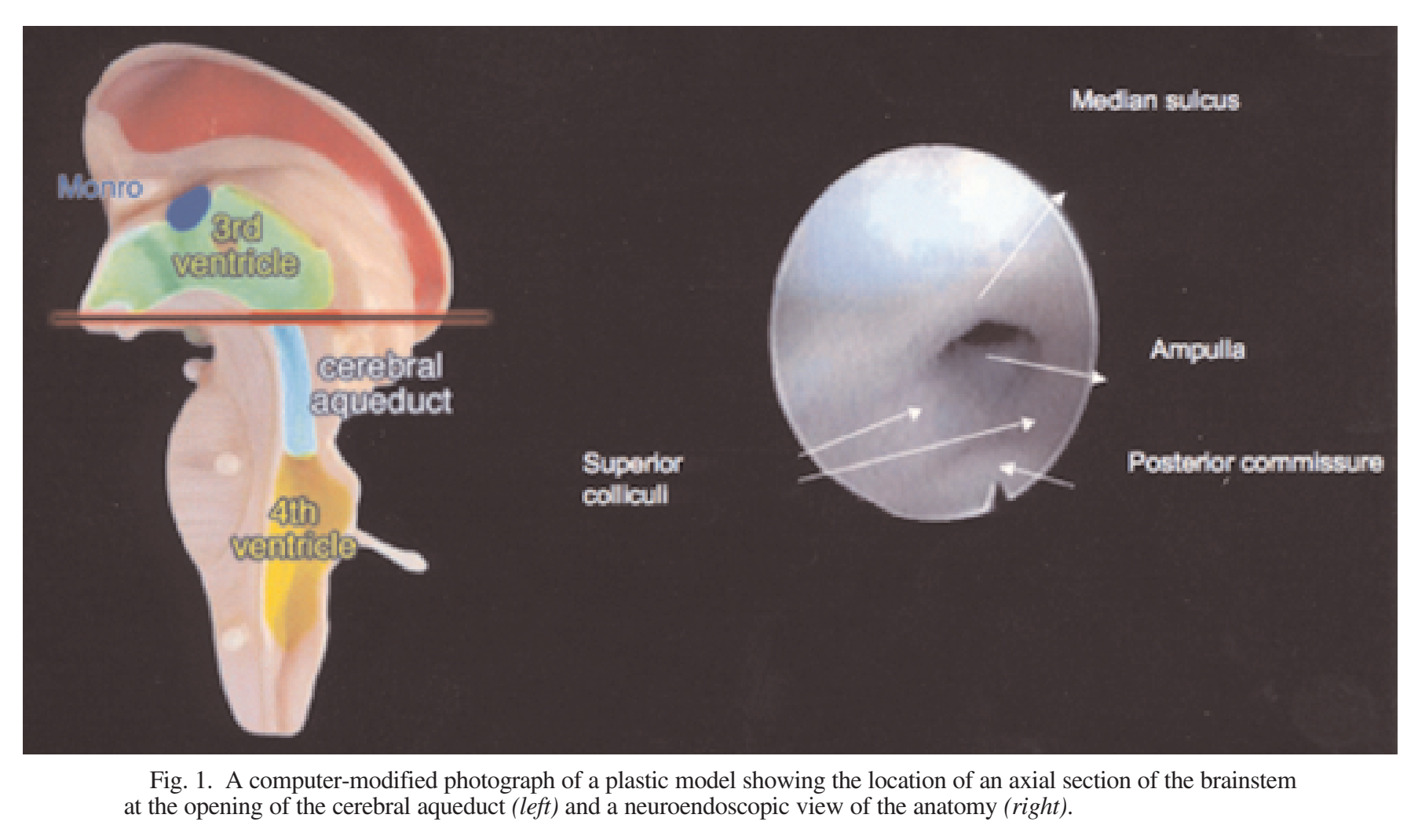

scriptorius and the foramen of Magendie (Fig. 3). In the fourth ventricle, the sulcus medianus acts as a route to the calamus scriptorius, but generally the instrument's advancement causes a physiological deflection toward the contralateral left lateral recess, then to the left foramen of Luschka. Navigation of the posterior triangle of the rhomboid fossa is possible, although it is anatomically delimited by the striae medullares ventriculi quarti. Nevertheless, it is sufficient to rotate the endoscope until crossing the midline, then reaching toward the calamus scriptorius and the obex above, which contain the canalis centralis medullaris spinalis.

Cerebellar tonsils and posterior inferior cerebellar arteries surround and delineate the dorsal part of the foramen of Magendie, which, when patent and wide, allows a view to the posterior oblongata and posterior part of the foramen magnum outside the fourth ventricle. Inspection of the foramen of Luschka is obtained by simply rotating the endoscope a little with moderate flexion. When withdrawing the scope, it is always advisable to look into the opening of the cerebral aqueduct to notice possible signs of small ependymal contusions.

\section{PATIENT SERIES}

More than 300 endoscopic procedures have been performed between 1994 and 2005 at our institution, and these procedures include 54 endoscopic navigations of the fourth ventricle. We reviewed our cases by retrieving data from both our neuroendoscopic clinical and video databases. The clinical series was subdivided into six clinicopathological groups, as follows. Group 1, five patients with a trapped fourth ventricle who underwent aqueductoplasty and stent insertion, followed by exploration of fourth ventricle outlets. Group 2, 19 patients harboring apparently communicating normal-pressure hydrocephalus treated with endoscopic third ventriculostomy, in whom navigation of the fourth ventricle was performed to rule out possible closing of outlets. Group 3, five patients with triventricular hydrocephalus due to membranous occlusion of the fourth ventricle. Group 4, 20 patients with intraventricular hemorrhages who underwent endoscopic ventricular clot aspiration, including the fourth ventricle. Group 5, three cases of aqueductal exophytic soft gliomas floating toward the fourth ventricle, which were directly aspirated. Group 6, two cases (one colloid cyst and one cystic intraventricular craniopharyngioma) in which the fourth ventricle was explored to aspirate fragments of colloid or cholesteremic cyst contents.

Our series is heterogeneous, but we tried to identify the difficulties and the feasibilities of the technical procedure for each case reviewed. Neuroendoscopic navigation of the fourth ventricle was successfully performed in all cases; we recorded four episodes of extreme bradycardia, and small ependymal contusions of the opening of the cerebral aqueduct in two cases. Possible brain damage due to navigationrelated injury is difficult to see in the neuroendoscopic assessment of intraventricular hemorrhages because of the presence of clots; besides, in chronic hydrocephalus the aqueduct could be so dilated that no resistance is offered to the introduction of an endoscope.

\section{DISCUSSION}

Authors of few reports have focused on the specific issue 


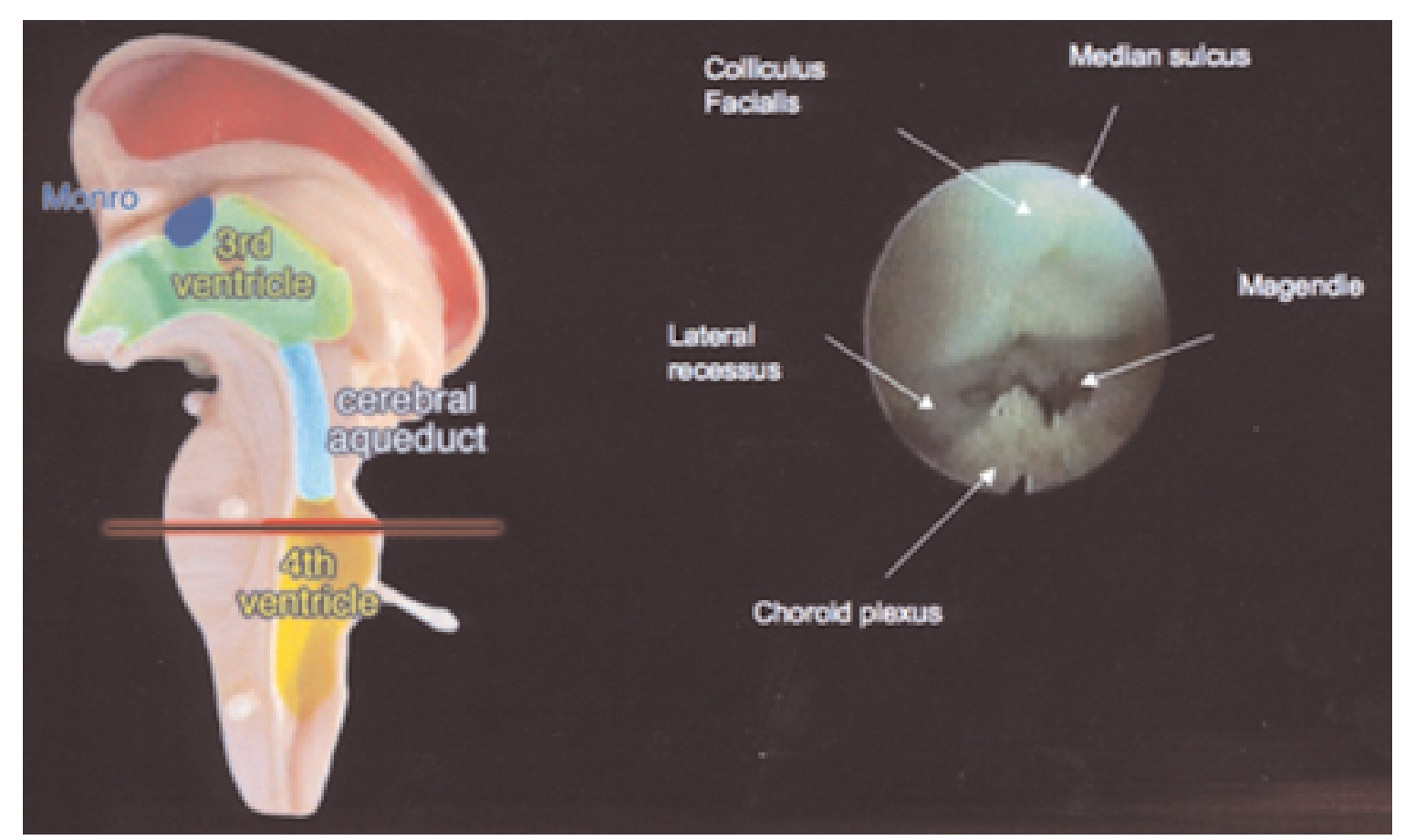

Fig. 2. Specimen showing the location of an axial section of the brainstem at the superior triangle of the rhomboid fossa in the fourth ventricle (left) and neuroendoscopic visualization of the ventricular anatomical structures (right).

of endoscopic exploration of the fourth ventricle. Matula, et al., ${ }^{7}$ stated that there are three possible ways to reach the fourth ventricle endoscopically: 1) coming from the third ventricle via the cerebral aqueduct; 2) through the basal cistern opening of the lateral foramen of Luschka; and 3) via the cerebellomedullary cistern through the foramen of $\mathrm{Ma}$ gendie.

The history of neuroendoscopic exploration of the fourth ventricle has been written by neurosurgeons using rigid endoscopes rather than by those adopting the more appropriate flexible ones. Endoscopic approaches to the aqueductal region via a frontal route were performed by Schroeder and Gaab, ${ }^{9}$ who used rigid endoscopes for the treatment of aqueductal stenosis in 17 patients. These authors observed postoperative sequelae mainly associated with disturbances of ocular movement.

Anandh, et al., ${ }^{1}$ described a right transfrontal approach in which a rigid endoscope was used to enter the lateral and third ventricle to remove fourth ventricle cysts in three patients. Cystic lesions were removed in all three with no deaths, although one patient experienced transient hemiparesis and ocular ptosis. In a patient with fourth ventricle neurocysticercosis, Proano, et al., ${ }^{8}$ positioned a rigid endoscope in front of the opening of the cerebral aqueduct and aspirated the cysts through a No. 6 French catheter.

Experience with the flexible endoscope for exploration of the fourth ventricle in five cases was reported by Bergsneider. ${ }^{3} \mathrm{He}$ avoided the transaqueductal pathway, however, and preferred a midline durotomy between the opisthion and the posterior arch of C-1, advancing toward the fourth ventricle through the foramen of Magendie.

Neuronavigation beyond the aqueduct can be of some interest in carefully selected situations. ${ }^{2}$ Our preliminary ex- periences commenced with cases of trapped fourth ventricles in infantile posthemorrhagic hydrocephalus. In these cases, the surgical action consisted of the shearing of the aqueductal membrane, which was further dilated with cautious partial inflation of a particular Fogarty balloon. Exploration proved to be very difficult because of the enlarged ventricle and poor visualization of the rhomboid fossa; in most of the cases this structure was so deformed that it had become unrecognizable.

Another interesting indication for fourth ventricle exploration is the total removal of intraventricular hemorrhages detailed in previous reports. ${ }^{6}$ What we learned is the ease of clearing clots from the fourth ventricle with this procedure compared with others. Also, the time spent varied greatly: a few minutes in the fourth ventricle and approximately 1 hour to clear each lateral ventricle. This unexpected ease in dealing with the fourth ventricle navigation was further confirmed by postoperative follow-up neuroimages, which revealed the fourth ventricle to be completely clot free. Once again, the anatomical integrity, when exiting the opening of the cerebral aqueduct, revealed no signs of damages related to the endoscopic maneuvers.

Many theoretical objections could be raised against the transaqueductal navigation of the fourth ventricle, but in light of our experience, this procedure was found to be relatively easy and harmless. The only neurological complication observed in our series was a mild diplopia in a patient in whom aqueductoplasty was followed by patency of the fourth ventricle outlets. The neurological deficit was almost certainly caused by the aqueductoplasty rather than by the transaqueductal passage of the endoscope.

The previously reported experiences of extreme bradycardia due to excessive irrigation ${ }^{5}$ have taught us to be 


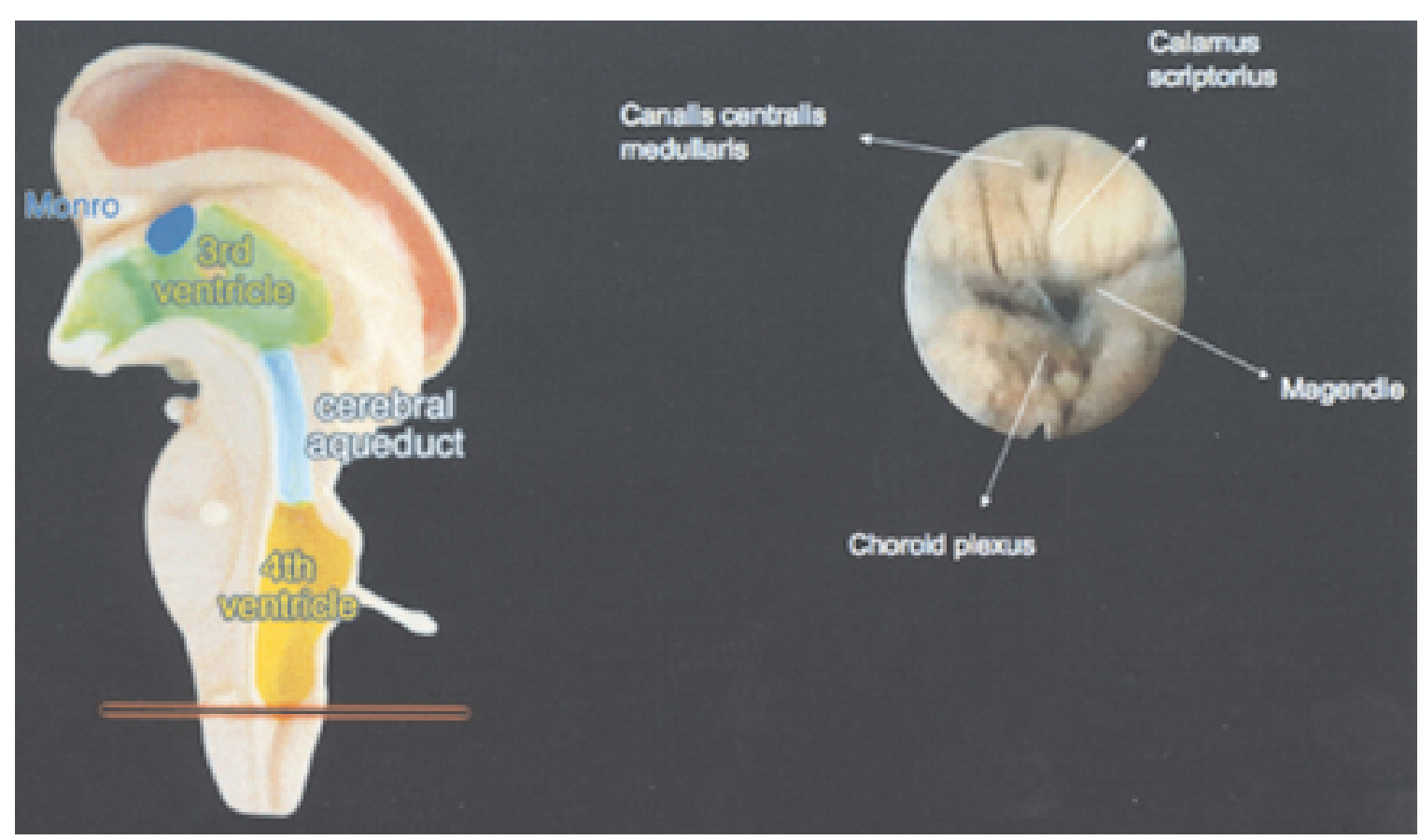

Fig. 3. Specimen showing the location of an axial section of the brainstem at the inferior triangle of the rhomboid fossa in the fourth ventricle (left) and neuroendoscopic visualization of the ventricular anatomical structures (right). Magendie $=$ foramen of Magendie.

much more cautious and accurate in dealing with the fourth ventricle and possible hypertension. Even if episodes of a significant bradycardia occurred, they immediately regressed after the withdrawal of small volumes of ventricular fluid.

\section{CONCLUSIONS}

We think that transaqueductal exploration of the fourth ventricle is feasible and reasonably safe, provided that the surgeon has extensive experience in neuroendoscopy performed using flexible instruments managed with the freehand technique.

\section{References}

1. Anandh B, Mohanty A, Sampath S, et al: Endoscopic approach to intraventricular cysticercal lesions. Minim Invasive Neurosurg 44:194-196, 2001

2. Barkhof F, Kouwenhoven M, Scheltens P, et al: Phase-contrast cine MR imaging of normal aqueductal CSF flow. Acta Radiol 35:123-130, 1994

3. Bergsneider M: Endoscopic removal of cysticercal cysts within the fourth ventricle. Technical note. J Neurosurg 91:340-345, 1999

4. Flyger G, Hjelmquist U: Normal variations in the caliber of the human cerebral aqueduct. Anat Rec 127:151-162, 1957
5. Handler MH, Abbott R, Lee M: A near-fatal complication of endoscopic third ventriculostomy: case report. Neurosurgery 35:525-528, 1994

6. Longatti PL, Martinuzzi A, Fiorindi A, et al: Neuroendoscopic management of intraventricular hemorrhage. Stroke 35:35-38, 2004

7. Matula C, Reinprecht A, Roessler K, et al: Endoscopic exploration of the IVth ventricle. Minim Invasive Neurosurg 39:86-92, 1996

8. Proano JV, Madrazo I, Avelar F, et al: Medical treatment for neurocysticercosis characterized by giant subarachnoid cysts. $\mathbf{N}$ Engl J Med 345:879-885, 2001

9. Schroeder HW, Gaab MR: Endoscopic aqueductoplasty: technique and results. Neurosurgery 45:508-518, 1999

10. Woollam DHM, Millen JW: Anatomical considerations in the aethiology of stenosis of the cerebral aqueduct. Brain 76: 104-112, 1953

Manuscript received October 20, 2005.

Accepted in final form November 21, 2005.

Address reprint requests to: Luca Basaldella, M.D., Divisione Clinicizzata di Neurochirurgia, Ospedale Regionale di Treviso, Ca' Foncello, Facoltà di Medicina e Chirurgia, Università degli Studi di Padova, Piazza le Ospedale Civile 1, 31100 Treviso, Italia. email: lbasaldella@ulss.tv.it. 